\title{
The balance of comprehensive coagulation and fibrinolytic potential is disrupted in patients with moderate to severe COVID-19
}

\author{
Tomoko Onishi ${ }^{1} \cdot$ Naruto Shimonishi ${ }^{1} \cdot$ Masahiro Takeyama ${ }^{1}$ (1) Shoko Furukawa ${ }^{1} \cdot$ Kenichi Ogiwara ${ }^{1}$. \\ Yuto Nakajima ${ }^{1} \cdot K_{\text {Kei Kasahara }}{ }^{2} \cdot \mathrm{Kenji} \mathrm{Nishio}^{3} \cdot \mathrm{Kiyomi} \mathrm{Yoshimoto}^{3} \cdot$ Satoki Inoue $^{4} \cdot$ Masahiko Kawaguchi $^{4}$. \\ Hidetada Fukushima ${ }^{5} \cdot$ Yoshihiko Saito $^{6} \cdot$ Hitoshi Yoshiji $^{7} \cdot$ Shigeo Muro $^{8} \cdot$ Kazuhiko Tsuruya $^{9} \cdot$ Sadanori Okada $^{10}$. \\ Kazuma Sugie $^{11}$. Ryuji Kawaguchi ${ }^{12} \cdot$ Toshiya Nishikubo $^{13} \cdot$ Masaharu Yamazaki $^{14} \cdot$ Yukio Oda $^{15} \cdot$ Toshiki Kawabe $^{15}$. \\ Kengo Onishi ${ }^{15} \cdot$ Tomohisa Nishio $^{15} \cdot$ Keiji Nogami $^{1}$
}

Received: 5 December 2021 / Revised: 7 February 2022 / Accepted: 7 February 2022 / Published online: 16 February 2022

(c) Japanese Society of Hematology 2022

\begin{abstract}
Coagulation and fibrinolytic mechanisms are enhanced in patients with coronavirus (COVID-19), but disturbances in the balance of both functions in COVID-19 patients remain unclear. We assessed global coagulation and fibrinolysis in plasma from 167 COVID-19 patients (mild/moderate/severe: 62/88/17, respectively) on admission using clot-fibrinolysis waveform analysis (CFWA). Maximum coagulation velocity $(|\mathrm{min} 1|)$ and maximum fibrinolysis velocity (IFL-min $1 \mid)$ were expressed as ratios relative to normal plasma. Ten patients $(6.0 \%)$ developed thrombosis, $5(3.0 \%)$ had bleeding tendency, and 13 (7.8\%) died during admission. FDP levels increased with severity of COVID-19 symptoms (mild/moderate/severe; median 2.7/4.9/9.9 $\mu \mathrm{g} / \mathrm{mL}$, respectively). The Imin1/ ratios were elevated in all categories (1.27/1.61/1.58) in keeping with enhanced coagulation potential, with significant differences between mild cases and moderate to severe cases. The IFL-min1I ratios were also elevated in all groups (1.19/1.39/1.40), reflecting enhanced fibrinolytic potential. These data identified coagulation dominance in moderate to severe cases, but balanced coagulation and fibrinolysis in mild cases. There were significant differences in FDP and TAT, but no significant differences in Imin1I or IFL-min1l ratios, between patients with and without thrombosis. CFWA monitoring of coagulation and fibrinolysis dynamics could provide valuable data for understanding hemostatic changes and disease status in COVID-19 patients.
\end{abstract}

Keywords COVID-19 $\cdot$ Coagulation $\cdot$ Fibrinolysis $\cdot$ Severity $\cdot$ Monitoring

\section{Introduction}

The novel coronavirus disease denominated COVID-19 is caused by infection with severe acute respiratory syndrome coronavirus 2 (SARS-CoV-2), and continues to be an evolving pandemic in the world. COVID-19 presents with high contagion, morbidity, and mortality. Since first reported in

Statement of prior presentation: An account of this work was presented at the 83th Annual Meeting of Japanese Society of Hematology 2021, Sendai, Japan.

Tomoko Onishi and Naruto Shimonishi contributed equally as the first author.

Masahiro Takeyama

mtake@naramed-u.ac.jp

Extended author information available on the last page of the article
December 2019, the virus has spread to over 200 countries and territories, infecting over 200 million people worldwide, and claiming the lives over 4 million people [1].

The SARS-CoV-2 spike protein interacts with both cellular heparan sulfate and angiotensin-converting enzyme 2 (ACE2) on the surface of alveolar endothelial cells through its receptor-binding domain [2-5]. This interaction governs the ability of SARS-Cov-2 virus to infect host cells. SARSCoV-2 infection induces a process known as immunothrombosis, in which activated neutrophils and monocytes interact with platelets in the coagulation cascade, leading to intravascular clot formation in small and larger vessels $[6,7]$. In severe cases, progression constitutes to the acute respiratory distress syndrome with severe endothelial damage and extensive thrombosis.

A high incidence of coagulation abnormalities and thrombotic complications is a prominent feature in patients with 
COVID-19. Fibrin deposition in the lung, elevated D-dimers, and thrombosis rates of 5-30\% despite thromboprophylaxis are characteristic of COVID-19-associated pneumonia [8-12]. Great attention is being paid to the causes, prevention and treatment of thrombosis in these circumstances.

Postmortem analyses of lungs from patients with COVID19 revealed the presence of fibrin in the pulmonary microvasculature and alveolar space [13], suggesting that fibrin deposition contributes to morbidity and mortality [13-15]. In addition, considerable hemorrhage as well as thrombosis was observed in the lungs [13]. Diagnosis and regulation of immunothrombosis and hemorrhage together with assessment of the coagulation-fibrinolysis balance may, therefore, help to prevent the progression of lung injuries associated with COVID-19. Disturbances of the coagulation and fibrinolysis systems have been identified in patients with COVID-19 [16-23]. However, while the coagulation mechanism has been extensively studied, the sequential fibrinolytic reactions have been less investigated. Furthermore, the extent of thrombus formation is regulated by a balance between coagulation and fibrinolysis, and this critical relationship remains to be comprehensively evaluated in patients with COVID-19.

Clot-fibrinolysis waveform analysis (CFWA) has been developed as a novel adaptation of the original CWA that was designed to assess global hemostatic mechanisms, and the modified technique concurrently monitors the potential for both clot formation and fibrinolysis [24]. In this context, we have recently determined the functional potentials of coagulation and fibrinolysis at diagnosis in patients with sepsis-associated disseminated intravascular coagulation (DIC) [25]. The CFWA parameters enabled classification of the hemostasis-related pathology of DIC at diagnosis into four types: coagulation-dominant, coagulation/fibrinolysisbalanced, fibrinolysis-dominant, and consumption-impaired coagulation, and the data indicated that most patients in our sepsis-associated DIC cohort were coagulation-dominant (80.7\%) [25]. Our findings indicated that comprehensive assessment of coagulation and fibrinolysis using CFWA in sepsis-associated DIC could be informative for in clinical practice. In COVID-19, a widespread inflammatory response and pulmonary immunothrombosis may govern the pathogenesis of abnormal coagulation and fibrinolysis confined to the lung [26]. This appears to be distinct from septic DIC, which causes marked systemic coagulation activity [27, 28]. Nevertheless, we considered that assessing the natural balance between coagulation and fibrinolysis could contribute to the development of a therapeutic strategy for preventing COVID-19-induced pulmonary damage and thrombus formation. The present study was designed, therefore, to examine global coagulation and fibrinolytic function using CFWA in patients with COVID-19 at admission to our hospital.

\section{Materials and methods}

\section{Ethical consideration}

This study was approved by the Medical Research Ethics Committee of Nara Medical University (No. 2705).

\section{Reagents}

The activated partial thromboplastin time (APTT) reagent (Coagpia\#APTT-N $)^{1}$ was obtained from SEKISUI MEDICAL CO., LTD., (Tokyo Japan). Recombinant tissue-type plasminogen activator (r-tPA; Activacin®; specific activity $580,000 \mathrm{IU} / \mathrm{mg}$ ) was purchased from American Diagnostica Inc. (Stamford, CT, USA). Other reagents are identified in relevant parts of the text.

\section{Patients}

Patients with COVID-19 who were admitted to Nara Medical University Hospital during August 3rd 2020 to February 28th 2021 participated in the study. Patients that have received anticoagulants that affect the APTT (e.g., warfarin, dabigatran etexilate, etc.) were precluded. Blood samples were obtained from 167 individuals at the time of admission. All blood samples were obtained initially for clinically essential coagulation studies, and suitable aliquots were subsequently utilized after informed consent from patients or legal representatives following the ethical guidelines of Nara Medical University. The clinical severity of COVID19 was defined as follows; (i) those who did not require any oxygen support, mechanical ventilation, and extracorporeal membrane oxygenation (ECMO) were considered as mild, (ii) patients who required supplementary oxygen support were categorized as moderate, and (iii) those who required mechanical ventilation, ECMO, or admitted to intensive care unit were regarded as severe.

\section{Plasma samples}

Whole blood samples $(1.8 \mathrm{~mL})$ were obtained using evacuated anti-coagulant tubes containing a $1: 9$ volume of $3.2 \%$ $(w / v)$ trisodium citrate. After centrifugation for $15 \mathrm{~min}$ at $1500 \times g$, the plasmas were stored at $-80^{\circ} \mathrm{C}$, and thawed at $37^{\circ} \mathrm{C}$ immediately prior to assay. Normal plasmas were collected from 20 normal healthy individuals (14 males and 6 females; ages ranging from 23 to 49 years) after informed consent following the local ethical guidelines.

\footnotetext{
1 "Coagpia" and "Nanopia" are trademark or registered trademark of SEKISUI MEDICAL CO., LTD. in Japan and/or other countries.
} 
(a)

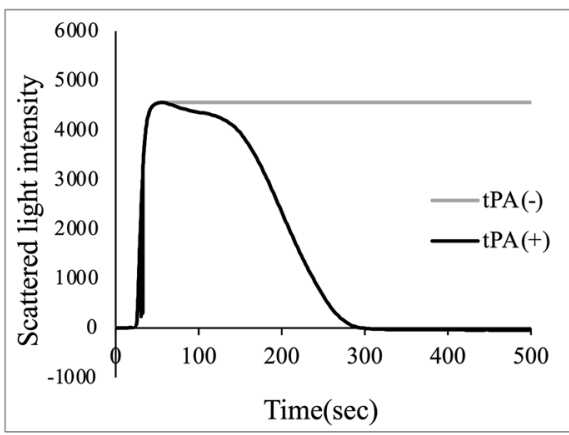

(b)

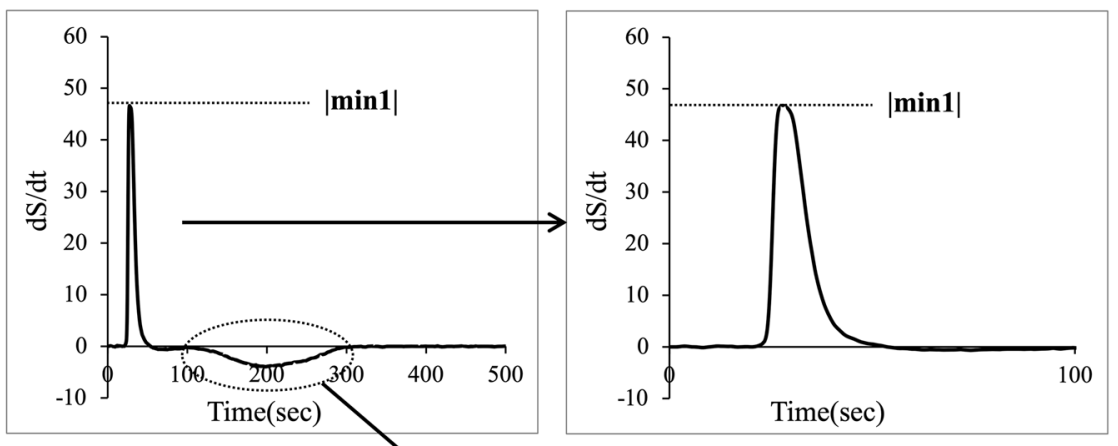

(d)

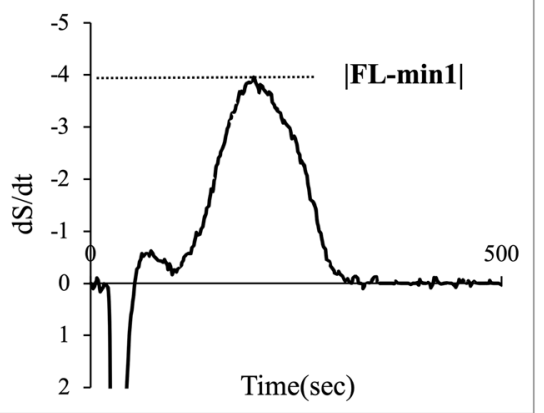

Fig. 1 Clot-fibrinolysis waveforms and parameters in normal plasma. Normal plasma was mixed with the APTT reagent, before the addition of $\mathrm{CaCl}_{2}$ together with r-tPA $(0.63 \mu \mathrm{g} / \mathrm{mL})$. a Shows the changes in scattered light intensity (S) observed over time during the performance of the APTT. b Illustrates the curves of the first deriva-

\section{Conventional laboratory parameters for coagulation and fibrinolysis}

The plasma levels of fibrinogen and antithrombin, fibrinogen/fibrin degradation products (FDP) and D-dimer, were measured using commercially available reagents. Plasmin activator inhibitor 1 (PAI-1), thrombin-antithrombin complex (TAT) and plasmin-antiplasmin complex (PIC), were measured using Nanopia\#PAI-1, Nanopia\#TAT (SEKISUI MEDICAL CO., LTD., Tokyo, Japan) and Lias Auto PIC (Sysmex Corp., Kobe, Japan).

\section{Simultaneous clot-fibrinolysis waveform analysis (CFWA)}

CFWA was performed on a CP3000 3 TM apparatus (SEKISUI MEDICAL) to monitor the dynamic processes of fibrin formation and clot lysis as reported earlier [24, 29] with minor modifications. [24, 29]. For patients on anticoagulation with heparin, heparinase (hep-TEM®Lyo, Finggal link, Munich, Germany) dissolved in $40 \mathrm{mM}$ HEPES Buffer ( $\mathrm{pH}$ 7.35) was added to the plasma to counteract its effect prior to tive over time (dS/dt) of the waveform shown in (a). c Illustrates an enlarged image of the curve during the coagulation phase shown in (b). d Illustrates an enlarged image of the inverted curve during the fibrinolysis phase (indicated by the dotted circle) in (b). |min $1 \mid$ maximum coagulation velocity, $\mid F L$-min $1 \mid$ maximum fibrinolysis velocity

measurement. Briefly, plasma samples $(50 \mu \mathrm{L})$ were mixed with activated APTT reagent $(50 \mu \mathrm{L})$, followed by the addition of solution containing $25 \mathrm{mM} \mathrm{CaCl}_{2}(50 \mu \mathrm{L})$ together with optimized concentrations of r-tPA (f.c. $0.63 \mu \mathrm{g} / \mathrm{mL}$; $365.4 \mathrm{IU} / \mathrm{mL}$ ). The clot-fibrinolysis waveform raw data were used for the analysis. The reaction time (seconds) was plotted on the horizontal axis, and scattered light intensity, determined at a wavelength of $660 \mathrm{~nm}$ during the whole course, was plotted on the vertical axis (Fig. 1a). The first derivative of scattered light intensity $(d S / d t)$ reflected the coagulation and fibrinolysis velocities (Fig. 1b). The maximum value of the first derivative (min1) was calculated as an indicator of the maximum velocity of coagulation (Fig. 1c). In this later phase (Fig. 1b), the data generated an inverted curve, and the maximum velocity of fibrinolysis (IFL-min1l) was determined from this curve (Fig. 1d). The parameters (Imin1I, |FL-min 1|) were further calculated as ratios relative to those obtained from normal plasma. The coagulation-fibrinolysis balance was indicated by the difference between the comprehensive coagulation and fibrinolysis potential ( $|\min 1|$ ratio-|FL-min 1/ ratio), and when the coagulation potential is greater than fibrinolysis potential $(|\min 1|$ ratio $>\mid$ FL-min1| 
ratio), we defined as coagulation-dominant. Calculations were performed using Excel software.

\section{Study population}

Clinical variables including demographics, comorbidities, platelet count, prothrombin time (PT), APTT, antithrombin, fibrinogen, FDP, and D-dimer at the time of admission were obtained from medical records.

\section{Data analysis}

Data are presented as median [interquartile range; IQR]. Association between clinical characteristics and severity were assessed by a logistic regression analysis. Differences between groups were compared by the Wilcoxon rank-sum test, and the correlations between parameters were analyzed using the Spearman correlation coefficient $(\rho)$. Multiple comparisons among different patient groups were determined by Kruskal-Wallis one way analysis of variance. $p$ values of $<0.05$ were considered as statistically significant. All statistical analyses were conducted using JMP 10.0.2 (SAS Institute Inc., Cary, NC).

\section{Results}

\section{Baseline clinical characteristics of patients}

One hundred and sixty-seven patients that had been diagnosed with COVID-19 (91 males, 76 females) were included. Clinical characteristics are summarized in Table 1. The median age [IQR] was 71 years [58-80], and 62 patients were characterized as mild, 88 were moderate, and 17 were severe. One hundred and seven patients (64.0\%) had one or more coexisting medical condition, of which systemic hypertension (44.9\%) and diabetes mellitus (25.7\%) was the most common. Twenty-nine patients (17.4\% of the total) had underlying cardiovascular disease. The severity of COVID19 was associated with systemic hypertension (odds ratio; mild vs. moderate 2.37 , mild vs. severe 4.15$)(p<0.05)$, and four of the moderate patients (4.5\%) were obese, which was less than that reported outside Japan [30, 31].

\section{Conventional laboratory data and clinical severity in COVID-19 patients}

The relationship between the common laboratory data of coagulation and fibrinolytic function and the clinical severity of COVID-19 at the time of admission are shown in Fig. 2. The basic coagulation parameters, APTT and PT, tended to prolong in association with disease severity, suggesting reduced coagulation potential. Fibrinogen and TAT levels

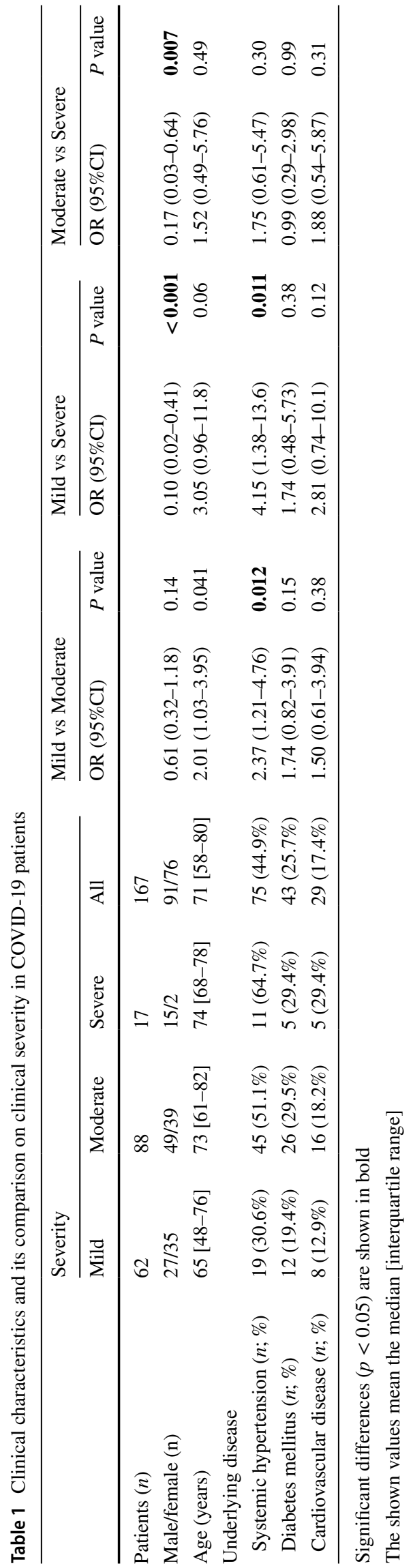



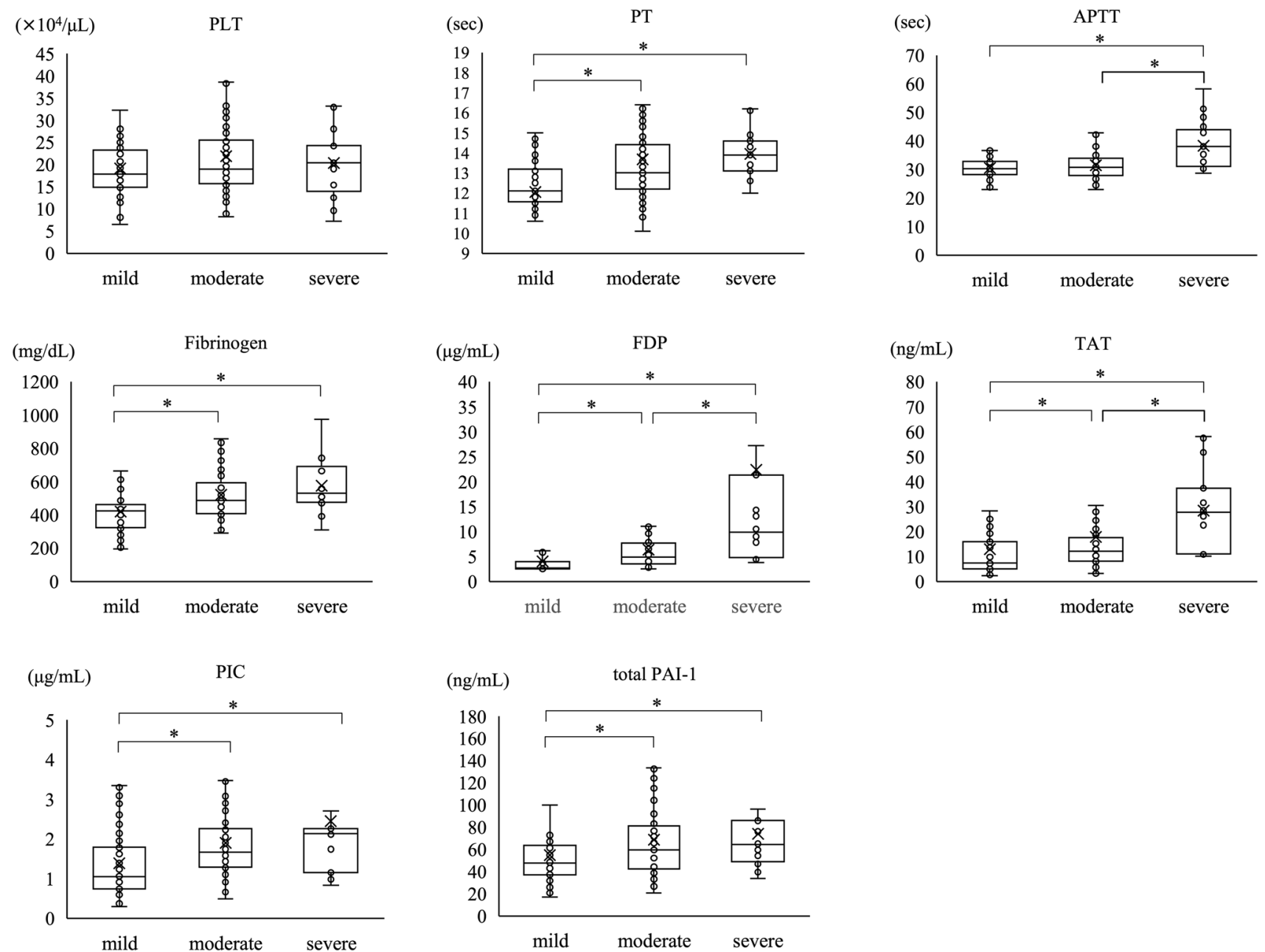

Fig. 2 Relationships between laboratory markers and clinical severities in COVID-19 patients. Relationships between laboratory markers at admission and clinical severities in COVID-19 patients are shown, together with boxplots of parameters in mild, moderate, and severe

were increased in all severities, however, and these measurements were higher in moderate and severe cases compared with those in mild cases $(p<0.05)$. These findings appeared to indicate that coagulation reactions in moderate and severe cases were more pronounced than in mild cases.

In addition, the fibrinolysis parameters, FDP and D-dimer, appeared to be related to disease severity $(p<0.05)$, although these specific assays were not covered by government health insurance in Japan at the time, and the number of patients available for these analyses was restricted. Consequently, these data were not included in the statistical analyses of our severe patients. The other fibrinolytic measurements, including PIC and total PAI-1 were higher than normal in all severities, and were more elevated in the moderate and severe categories than in mild cases $(p<0.05)$. Overall, therefore, it seemed to be difficult to derive firm conclusions regarding complete hemostatic status using these severity. The median values of parameters are depicted within the boxes. The boxes end at the 25 th and 75 th percentiles, and the whiskers extend to the furthest points that are not outliers. ${ }^{*} p<0.05$; significant difference

conventional coagulation and fibrinolysis tests in COVID-19 patients, although our results tended to suggest increased levels of coagulation, fibrinolysis, and anti-fibrinolysis function in moderate to severe patients relative to mild cases.

\section{Assessments of comprehensive coagulation and fibrinolysis potential in COVID-19 patients}

The CFWA technique is a straightforward procedure that provides a direct and comprehensive evaluation of fibrin clot formation and fibrinolytic potential [24, 25]. We utilized this technique, therefore, to assess these global interactions in COVID-19 patients. Representative clot-fibrinolysis waveforms in plasma samples obtained from patients with mild, moderate, and severe in severity are illustrated in Fig. 3a. Scattered light intensities were markedly elevated in relation to the degree of severity, and this enhanced comprehensive 
(a)

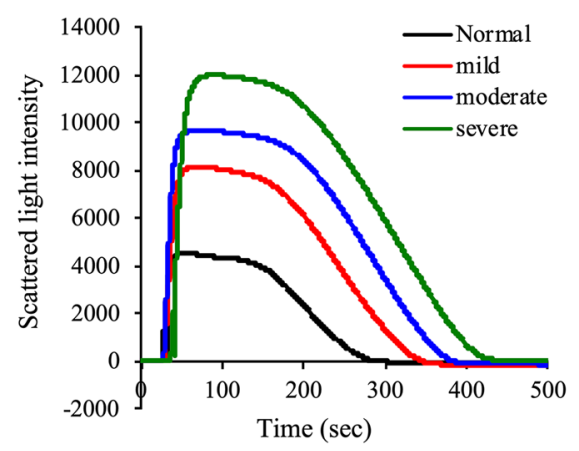

(b)

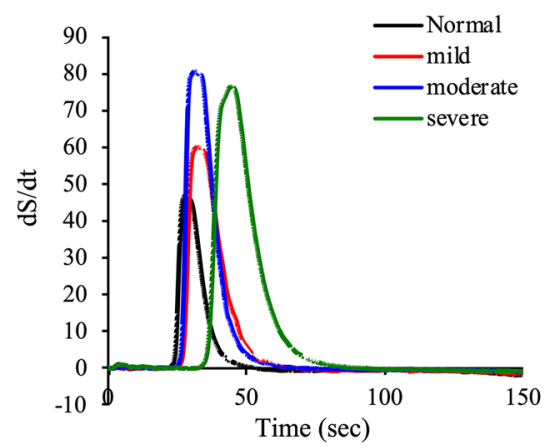

(c)

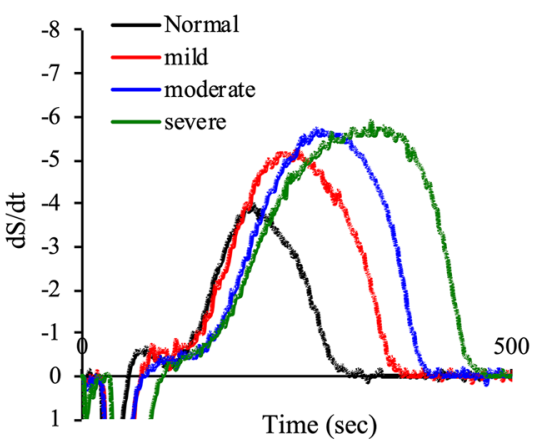

Fig. 3 Representative clot-fibrinolysis waveforms in COVID patients with various clinical severities. Normal (black) or patients' plasma samples (mild; red, moderate; blue, severe; green in severity) were mixed with the APTT reagent before the addition of $\mathrm{CaCl}_{2}$ together with r-tPA $(0.63 \mu \mathrm{g} / \mathrm{mL})$. a Shows the changes in scattered light intensity (S) observed over time during the performance of the APTT. b Illustrates enlarged images of the curves of the first derivative over time (dS/dt) during the coagulation phase of the waveforms shown in (a). c Illustrates enlarged images of the inverted curves of the first derivative over time $(d S / d t)$ during the fibrinolysis phase of the waveforms shown in (a)
Fig. 4 Relationships between CFWA parameters and clinical severities in COVID19 patients. The relationships of coagulation ( $|\min 1|$ ratio) and fibrinolysis parameters (IFL-min1I ratio) with clinical severity were assessed by CFWA in COVID-19 patients at admission to hospital. Boxplots of coagulation and fibrinolysis parameters in mild, moderate, and severe severity are shown. The median values of parameters are depicted within the boxes. The boxes end at the 25th and 75th percentiles, and the whiskers extend to the furthest points that are not outliers. ${ }^{*} p<0.05$; significant difference

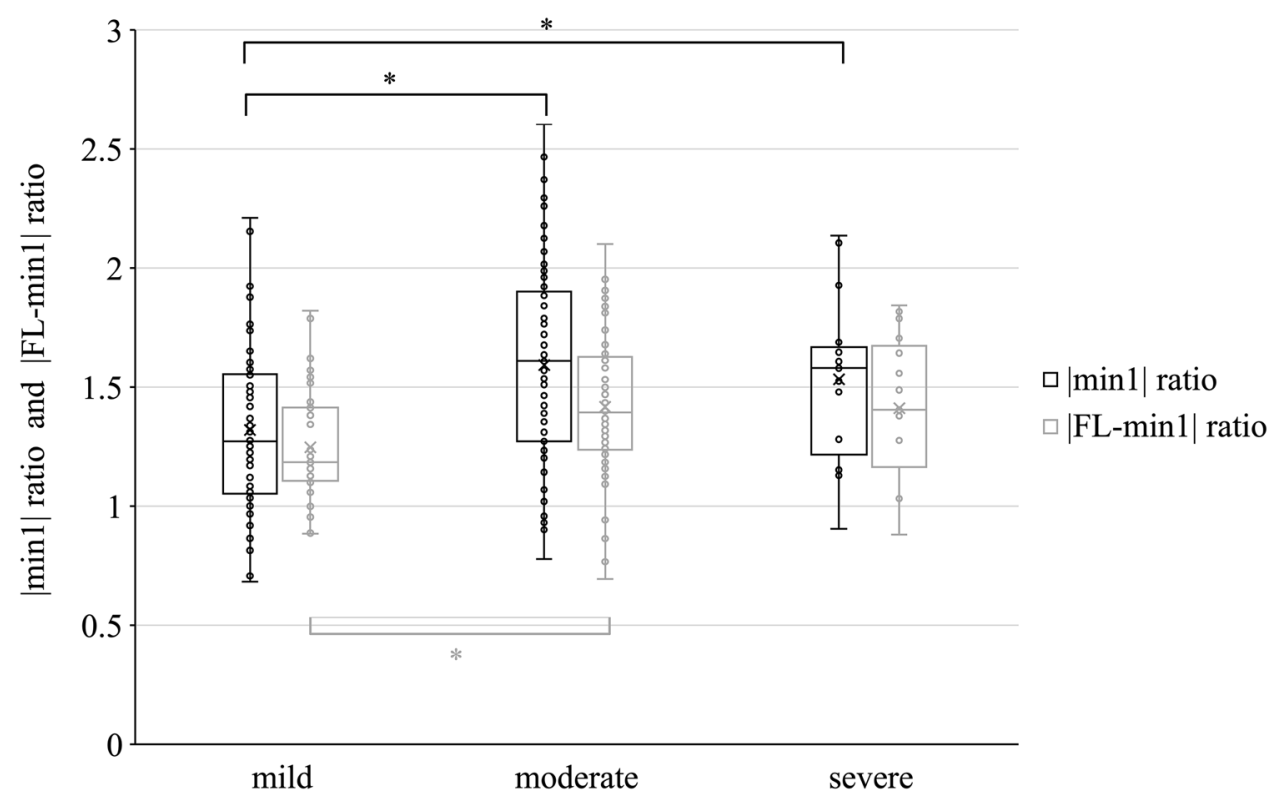

coagulation potential appeared to be accompanied by increased fibrinogen levels. Consequently, the calculated Imin1| values were markedly increased, especially in moderate and severe cases. Peak intensities returned to initial levels, regardless of clinical severity. In addition, the initiation of clot formation was somewhat delayed especially in severe cases (Fig. 3b), but the IFL-min1l was also increased relative to that in normal plasma, suggesting that fibrinolytic potential, was enhanced depending on the degree of severity (Fig. 3c).

The CFWA parameters were further analyzed as ratios relative to those obtained from normal plasma (Fig. 4, Supplemental Table 2). In all instances, the Imin1। ratios were increased, and demonstrated significant differences between mild (median [IQR]; 1.27 [1.05-1.56]) and moderate (1.61 [1.27-1.90]) cases, and between mild and severe cases (1.58 [1.22-1.67]), confirming that comprehensive coagulation potential was greater in moderate to severe patients compared to mildly affected cases. Moreover, IFL-min 1| ratios were also increased in all groups, and significant differences were evident between mild (1.19 [1.10-1.41]) and moderate (1.39 [1.23-1.63]) cases. Interestingly, the difference between the $I \min 11$ ratio and |FL-min1| ratio appeared to be greatest in patients with moderate severity. 
Table 2 Comparison with CFWA parameters and laboratory data in COVID-19 patients

\begin{tabular}{llcc}
\hline CFWA parameters & Laboratory data & $\begin{array}{c}\text { Correlation } \\
\text { coefficient }(\rho)\end{array}$ & $p$ value \\
\hline Imin1| ratio & PT $(\mathrm{sec})$ & 0.110 & 0.17 \\
& APTT $(\mathrm{sec})$ & -0.262 & $<\mathbf{0 . 0 0 1}$ \\
& TAT $(\mathrm{ng} / \mathrm{mL})$ & 0.279 & $<\mathbf{0 . 0 0 1}$ \\
& Fbg $(\mathrm{mg} / \mathrm{dL})$ & 0.606 & $\mathbf{< 0 . 0 0 1}$ \\
$\mid$ FL-min1| ratio & FDP $(\mu \mathrm{g} / \mathrm{mL})$ & 0.306 & $\mathbf{0 . 0 0 2 1}$ \\
& PIC $(\mu \mathrm{g} / \mathrm{mL})$ & 0.453 & $<\mathbf{0 . 0 0 1}$ \\
& Total PAI-1 $(\mathrm{ng} / \mathrm{mL})$ & 0.241 & $\mathbf{0 . 0 0 1 8}$ \\
\hline
\end{tabular}

Significant differences $(p<0.05)$ are shown in bold

The correlations between parameters were analyzed using the Spearman correlation coefficient $(\rho)$

$P T$ prothrombin time, FDP fibrinogen/fibrin degradation products, TAT thrombin-antithrombin complex, $P I C$ plasmin- $\alpha 2$-plasmin inhibitor complex, PAI-1 plasminogen activator inhibitor

\section{Relationship between CFWA parameters and clinical outcomes in COVID-19 patients}

Fifteen of our COVID-19 patients developed thrombotic or hemorrhagic complications, including deep venous thrombosis (DVT) in five cases, cerebral infarction in one case, and pulmonary embolism in one case, gastrointestinal bleeding in two cases and intracranial bleeding in three cases. In addition, 13 patients died during hospital admission. (Table 3 ). The incidence of mortality or thrombosis was higher in severe cases ( $17.6 \%$ and $23.5 \%$, respectively) relative to moderate cases (10.2\% and $4.5 \%$, respectively) and mild (1.6\% and $3.2 \%$, respectively). Although bleeding episodes were reported in all groups, comparisons were unreliable with the small numbers of patients recorded in each group.

Further analysis of this relationship between CFWA parameters and clinical outcome in COVID-19 patients (Table 4), demonstrated that there were significant differences in FDP

Table 3 Coagulopathy and clinical outcome in COVID-19 patients

\begin{tabular}{|c|c|c|c|c|}
\hline & Mild $(n=62)$ & Moderate $(n=88)$ & Severe $(n=17)$ & All $(n=167)$ \\
\hline \multicolumn{5}{|l|}{ Coagulopathy } \\
\hline Thrombosis & $2(3.2 \%)$ & $4(4.5 \%)$ & $4(23.5 \%)$ & $10(6.0 \%)$ \\
\hline Pulmonary embolism & & & 1 & 1 \\
\hline Deep venous thrombosis & 2 & 2 & 1 & 5 \\
\hline Cerebral infarction & & & 1 & 1 \\
\hline Arteriosclerosis obliterans & & & 1 & 1 \\
\hline Acute myocardial infarction & & 1 & & 1 \\
\hline Dialysis arteriovenous shunt occlusion & & 1 & & 1 \\
\hline Bleeding & $2(3.2 \%)$ & $2(2.3 \%)$ & $1(5.9 \%)$ & $5(3.0 \%)$ \\
\hline Gastrointestinal bleeding & & 1 & 1 & 2 \\
\hline Intracranial bleeding & 2 & 1 & & 3 \\
\hline Mortality rate $(n ; \%)$ & $1(1.6 \%)$ & $9(10.2 \%)$ & $3(17.6 \%)$ & $13(7.8 \%)$ \\
\hline
\end{tabular}

\section{Relationship between CFWA parameters and conventional laboratory parameters}

Comparisons between CFWA parameters and the common laboratory parameters are illustrated in Table 2. The Imin $1 \mid$ ratio correlated with APTT $(\rho=-0.262)$, TAT $(\rho=0.279)$, and, in particular fibrinogen levels $(\rho=0.606)$, but little correlation was apparent with PT. The IFL-min1| ratios correlated with all fibrinolytic parameters including FDP $(\rho=0.306)$, total-PAI-1 $(\rho=0.241)$, and appeared to be especially associated with PIC $(\rho=0.453)$. These results supported the use of CFWA for the clinical assessment of global hemostasis in COVID-19 patients. and TAT between the thrombosis group and non-thrombosis group ( $p=0.016, p=0.038$, respectively), but that there were no significant differences in Imin $1 \mid$ or $|F L-m i n 1|$ ratios related to thrombotic complications. Moreover, no significant differences between the survival group and non-survival group were evident with any of the laboratory assays.

\section{Discussion}

The present study describes, for the first time, the investigation of comprehensive coagulation and fibrinolysis potential using CFWA in COVID-19 patients at the time of hospital 
Table 4 Relationship between CFWA parameters with clinical outcomes in COVID-19 patients

\begin{tabular}{|c|c|c|c|c|c|c|}
\hline & \multicolumn{2}{|l|}{ Mortality } & \multirow[t]{2}{*}{$p$ value } & \multicolumn{2}{|l|}{ Thrombosis } & \multirow[t]{2}{*}{$p$ value } \\
\hline & Survived $(n=154)$ & Non-survived $(n=13)$ & & Absence $(n=157)$ & Presence $(n=10)$ & \\
\hline \multicolumn{7}{|l|}{ CFWA parameters } \\
\hline$|\min 1|$ ratio & $1.48[1.20-1.75]$ & $1.48[1.11-1.78]$ & 0.83 & 1.48 [1.19-1.75] & $1.29[0.99-1.76]$ & 0.39 \\
\hline |FL-min $1 \mid$ ratio & 1.35 [1.16-1.54] & $1.36[1.08-1.55]$ & 0.97 & $1.36[1.16-1.54]$ & $1.28[1.17-1.66]$ & 0.78 \\
\hline \multicolumn{7}{|l|}{ Laboratory data } \\
\hline $\mathrm{PT}(\mathrm{sec})$ & $12.9[12.0-14.0]$ & $13.0[12.1-14.9]$ & 0.57 & $12.9[12.0-14.0]$ & $13.4[11.5-15.1]$ & 0.51 \\
\hline APTT (sec) & $30.9[28.7-34.0]$ & 30.5 [27.9-37.4] & 0.48 & $30.7[28.6-34.0]$ & 33.9 [29.6-38.4] & 0.23 \\
\hline FDP $(\mu \mathrm{g} / \mathrm{mL})$ & $4.0[2.8-7.3]$ & $6.2[3.8-13.1]$ & 0.12 & $4.0[2.9-6.7]$ & $10.5[5.3-20.0]$ & 0.016 \\
\hline Fbg (mg/dL) & 464 [379-564] & 440 [390-511] & 0.85 & 461 [378-562] & 519 [421-650] & 0.34 \\
\hline TAT (ng/mL) & 11.3 [6.6-18.6] & $11.8[10.0-25.1]$ & 0.35 & $11.2[6.6-17.5]$ & $25.0[9.2-31.0]$ & 0.038 \\
\hline $\mathrm{PIC}(\mu \mathrm{g} / \mathrm{mL})$ & $1.5[1.0-2.1]$ & $1.7[1.3-2.1]$ & 0.28 & $1.49[1.02-2.03]$ & $1.8[1.3-2.9]$ & 0.19 \\
\hline Total PAI-1 (ng/mL) & $52.2[38.9-72.5]$ & $47.6[42.6-66.1]$ & 0.79 & $51.9[38.9-72.4]$ & 59.4 [47.9-67.4] & 0.55 \\
\hline
\end{tabular}

Significant differences $(p<0.05)$ are shown in bold

The median [interquartile range] values are shown

Paired Wilcoxon rank-sum tests were used as statistical tests

$P T$ prothrombin time, APTT activated partial thromboplastin test, FDP fibrinogen/fibrin degradation products, Fbg fibrinogen, TAT thrombinantithrombin complex, PIC plasmin- $\alpha 2$-plasmin inhibitor complex, $P A I-1$ plasminogen activator inhibitor

admission. Our findings demonstrated that the parameters of global hemostasis were abnormal in the majority of these patients, regardless of the clinical severity. The data indicated that the hemostatic balance was maintained in mild cases but was disordered to be coagulation-dominant in moderate to severe cases. This divergence of coagulation and fibrinolytic reactions appeared to be most pronounced in moderate cases with a tendency towards a pro-thrombotic state in individuals presenting moderate to severe COVID19 symptoms. The results were in keeping with an observational study showing that the mortality rate in severe COVID-19 patients receiving anticoagulant treatment was lower than that in those without anticoagulant treatment (29.1\% and $62.7 \%, p<0.001)$ [32].

Coagulation potential assessed by PT or APTT was decreased in moderate to severe cases, and were discrepant with one assessed by Imin1I. This reason appeared to be due to the influence of increased fibrinogen levels. Fibrinogen is known as the soluble precursor of fibrin, the fundamental component of clot formation and stability. In addition, elevated levels of fibrinogen are procoagulant [33] and influence blood viscosity. Consequently, elevated fibrinogen levels are common in COVID-19, and in view of these multi-functional roles, the association between increased fibrinogen concentrations and high Imin1/ ratios was expected. On the other hand, too much amount of fibrinogen antigen leads to a decrease of the waveform (the rate of permeation) and the prolongation of the initiation of clotting time. An earlier study reported that the in vitro addition of fibrinogen to normal plasma prolonged the clotting time [24]. Our data suggested that a tendency to enhanced coagulation potential should be considered even in the prolongation of APTT.

As with Imin1l, high plasma level of fibrinogen can lead to the high |FL-min1I. When exogenous fibrinogen is added in vitro to normal plasma, IFL-min1I appears to be elevated dose-dependently. However, the $\operatorname{lmin} 1 \mathrm{l}$ is also increased and the overall balance appears to be maintained [24]. Therefore, an elevation of IFL-min1I may indicate an increased fibrinolytic potential, but it would be important to evaluate both coagulation and fibrinolysis to determine if the overall balance is tilted toward fibrinolysis or if the fibrinolysis potential is truly increased, thus the obtained data may need to interpret carefully.

The pathogenesis of infection-associated thrombosis in COVID-19 appears to contrast with that of sepsis-associated DIC. The disseminated coagulopathy is induced by multiple microthrombi, and is characterized by markedly enhanced, systemic coagulation together with severely suppressed fibrinolytic potential $[25,34]$. Our findings revealed the presence of enhanced coagulation potential but non-suppressed fibrinolysis in COVID-19 cases. Severely defective coagulation function was observed in few patients. In addition, postmortem analyses of COVID-19 patients revealed the presence of thrombosis in the lungs, but no thrombus was found in other organs such as kidney, spleen, pancreas, and liver [13]. In SARS-CoV-2 infection, publications have reported that viral RNA was undetectable in the blood [35] or was detected in only $6(15 \%)$ of 41 patients [36], again arguing for a pathology centered around pneumocytes and adjacent tissue pathology, rather than systemic viral infection. Hence, the COVID-19-associated coagulopathy could 
Fig. 5 Distribution of coagulation and fibrinolytic potentials based on the parameters obtained by CFWA in COVID19 patients with various severities. Coagulation and fibrinolytic potentials were determined based on the parameters obtained by CFWA in COVID19 patients with various severities. The vertical axis shows the values calculated for the Imin $1 \mid$ ratio. The horizontal axis shows the analogous values for the |FL-min1| ratio. The diagonal line depicting unity ( $|\min 1|$ ratio $=\mid$ FL-min $1 \mid$ ratio $)$ is shown (dashed line). Panel (a) shows the distribution of coagulation and fibrinolytic potentials of all COVID-19 patients, and Panel (b), (c), and (d) show the distribution of patients with mild, moderate, and severe disease, respectively
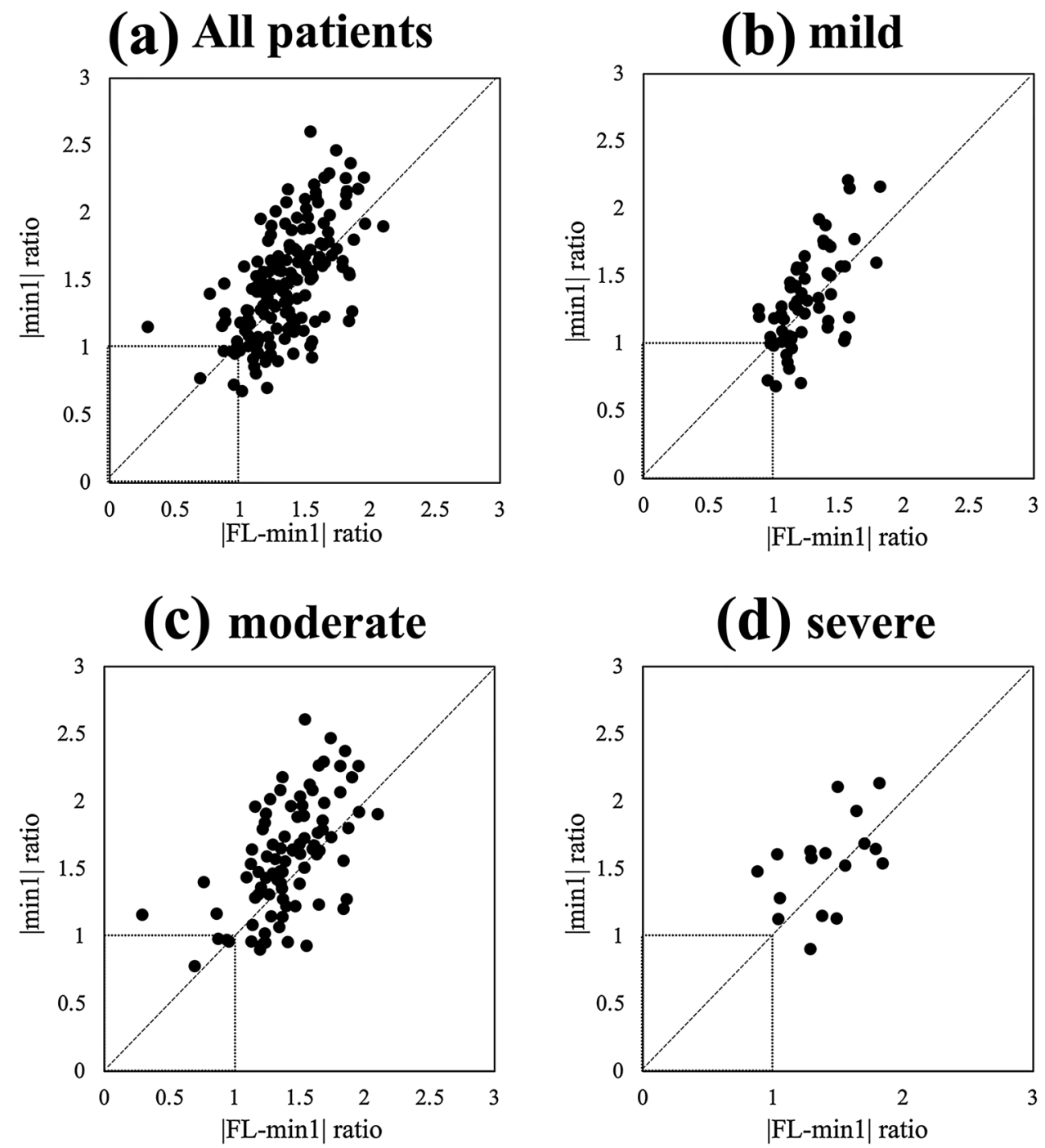

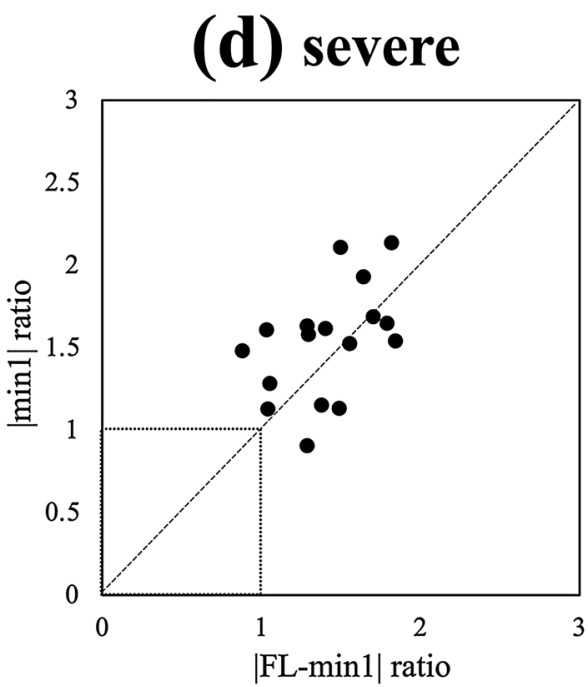

have been promoted by relatively localized cellular interactions, especially at sites of pulmonary vascular interfaces where the pathology is strongly manifested as organ damage.

There were no significant differences in any of our parameters between those patients that died from infection and those that survived. The risk of death due to hemostasis-related complications at the time of admission was difficult to determine, therefore, and continued evaluation of coagulation and fibrinolysis over time could be more informative. Significant differences in FDP and TAT were observed between the thrombotic and nonthrombotic groups, although no significant differences in $|\min 1|$ or $|F L-m i n 1|$ ratios were determined in these patients. A meta-analysis of thrombosis in COVID-19 patients reported that the incidence of venous thromboembolism (VTE) was $21 \%$ in all cases, $5 \%$ in general ward cases, and $31 \%$ in ICU cases [10], but the incidence of VTE in our ICU cases was low at 5.9\% (1/17). Difficulties with determining the clinical diagnosis of pulmonary thrombosis and DVT may partially explain, therefore, the lack of definitive laboratory data in our cohort. Nevertheless, the Imin1I ratio in our thrombosis group tended to be lower than that in non-thrombosis group, although the difference was not significant. Also, the Imin $1 \mid$ ratios were elevated in all severities of COVID-19 at the time of admission. This parameter appeared to be most elevated in moderate cases, and. tended to be lower in severe cases. It seemed possible, therefore, that a decrease in the Imin $1 \mid$ ratio over time may indicate possible developing thrombotic complications or worsening of the disease.

We have recently proposed a novel classification of DIC, based on the coagulation-fibrinolysis balance in patients with sepsis-associated DIC [25]. As shown in Fig. 5A, the CFWA results in our COVID-19 patients at admission supported the presence of both hyper-coagulation $(|\min 1|$ ratio $>1)$ and hyper-fibrinolysis (IFL-min1| ratio $>1$ ), suggesting a different pathogenesis from that in sepsis-associated DIC [25, 33]. These data again indicated that the hemostatic balance 
tended to deviate towards a coagulation-dominant status in patients with moderate COVID-19 severity (Fig. 5B-D).

Overall, therefore, thrombotic events were diagnosed in all categories of COVID-19 severity, suggesting that enhanced coagulation potential might be a risk factor for thrombosis even in the presence of increased fibrinolytic activity. In particular, thrombosis tended to be more common in severe cases, possibly associated with aggravating risk factors in circumstances where clinically worse patients are sedated and receive respiratory ventilation. Some bleeding symptoms were also seen in all groups of patients, emphasizing the importance of evaluating both coagulation and fibrinolysis function in these patients prior to therapeutic intervention.

We also examined the influence of anticoagulant therapy on thrombosis development in COVID-19 patients. The anticoagulant therapy performed in our hospital was only heparin administration. During their clinical courses, anticoagulant therapy was performed in $8.0 \%$ of mild cases, $25.0 \%$ of moderate cases, and $76.4 \%$ of severe cases, with the percentage of treatment increasing with disease severity. The number of patients who received anticoagulant therapy by severity and whether or not anticoagulant therapy was involved in thrombosis development are shown below (Supplemental Table 3). In this study, we could not evaluate the involvement of heparin treatment for the development of thrombosis because the number of patients who developed thrombosis was small and many of severe cases were treated with heparin administration.

Some limitations are evident in the present study, however. First, estimates of thrombosis and mortality rates could be dependent on cohort size, and the number of patients available for the current investigations was relatively small especially in severe cases. In addition, the present study included only patients at the time of admission to hospital, and further investigations throughout the clinical course of COVID-19 infection could be informative. And, it might be better to compare with COVID-19 and non-COVID-19 patients to evaluate the difference between COVID-19 conditions and other infections. However, we could not compare with non-COVID-19 and COVID-19 patients in the present study because we did not have enough cases to match the conditions. We would like to investigate the difference between COVID-19 and non-COVID-19 patients in the future. Finally, the assessment of coagulation and fibrinolysis balance should be done carefully. The result obtained by CFWA is regarded as the degree to which the overall coagulation and fibrinolytic function deviates from normal, with the balance tending toward the greater deviation. However, it might be unclear what extent the difference is coagulation-dominant or fibrinolysis-dominant precisely. Nevertheless, our findings suggested that analysis of comprehensive coagulation and fibrinolysis by CFWA, when combined with conventional clinical tests, could be useful for assessing the hemostatic balance, and could provide valuable data for decisions on clinical therapy in COVID-19. Furthermore, CFWA utilizes readily available automated equipment with a simply modified, regular APTT reagent, and requires minimum special training and skills [24].

Supplementary Information The online version contains supplementary material available at https://doi.org/10.1007/s12185-022-03308-w.

Acknowledgements The authors thank all the patients and their family members. We thank for Ms. Hanako Shimo for excellent special technique (Nara Medical University).

Author contributions TO collected samples, performed experiments, analyzed the data, interpreted the data, made the figures, wrote the paper. NS performed experiments and analyzed the data. MT designed the research, collected samples, did experimental supports, analyzed and interpreted the data, made the figures, wrote the paper, and approved the final version to be published. SF collected samples and performed experiments. KO collected samples and analyzed the data. YN interpreted data. YO, TK, KO, and TN analyzed the data. KK, KN, KY, SI, MK, HF, YS, HY, SM, KT, SO, KS, RK, TN, and MY did clinical supports. KN designed the research, interpreted the data, wrote the paper, made the figures, and edit the manuscript.

Funding This work was partly supported by SEKISUI MEDICAL CO., LTD.

\section{Declarations}

Conflict of interest T.O., N.S., M.T., S.F., K.O., Y.N., and K.N. have received research support from SEKISUI MEDICAL CO., LTD., and K.O., N.S. and K.N. possess the pending patent relating to CWA. K.N. received honoraria from this company. Y.O., T.K., K.O., and T.N. were employees of SEKISUI MEDICAL. K.K., K.N., K.Y., S.I., M.K, H.F., Y.S., H.Y., S.M., K.T., S.O., K.S., R.K., T.N., and M.Y. have no conflict of interest.

\section{References}

1. Organization WH. Coronavirus (COVID-19) Dashboard. https:// covid19. who.int Aug 2021.

2. Clausen TM, Sandoval DR, Spliid CB, Pihl J, Perrett HR, Painter $\mathrm{CD}$, et al. SARS-CoV-2 infection depends on cellular heparan sulfate and ACE2. Cell. 2020;183(4):1043-57.e15. https://doi.org/ 10.1016/j.cell.2020.09.033.

3. Jingwen Y, Weihua J, Hua Y, John F, Xuehong S, Hong Q, et al. Heparan sulfate facilitates spike protein-mediated SARS-CoV-2 host cell invasion and contributes to increased infection of SARS-CoV-2 G614 mutant and in lung cancer. Front Mol Biosci. 2021;11(8): 649575. https://doi.org/10.3389/fmolb.2021.649575 (eCollection).

4. Hoffmann M, Kleine-Weber H, Schroeder S, Krüger N, Herrler T, Erichsen S, et al. SARS-CoV-2 cell entry depends on ACE2 and TMPRSS 2 and is blocked by a clinically proven protease inhibitor. Cell. 2020;181(2):271-80. https://doi.org/10.1016/j.cell.2020.02. 052.

5. Lu R, Zhao X, Li J, Niu P, Yang B, Wu H, et al. Genomic characterisation and epidemiology of 2019 novel coronavirus: implications for virus origins and receptor binding. 
Lancet. 2020;395(10224):565-74. https://doi.org/10.1016/S01406736(20)30251-8.

6. Aldo B, Alessandra V, Lorenzo D, Kimberly M, Dave LD, Benjamin WVT, et al. Endothelial dysfunction and immunothrombosis as key pathogenic mechanisms in COVID-19. Nat Rev Immunol. 2021;21(5):319-29. https://doi.org/10.1038/s41577-021-00536-9.

7. Joan L, Daniella S, Michael N. COVID-19, immunothrombosis and venous thromboembolism: biological mechanisms. Thorax. 2021;76(4):412-20. https://doi.org/10.1136/thora xjnl-2020-216243.

8. Klok FA, Kruip MJHA, vander Meer NJM, Arbous MS, Gommers DAMPJ, Kant KM, et al. Incidence of thrombotic complications in critically ill ICU patients with COVID-19. Thromb Res. 2020;191:145-7. https://doi.org/10.1016/j.thromres.2020.04.013.

9. Cui S, Chen S, Li X, Liu S, Wang F. Prevalence of venous thromboembolism in patients with severe novel coronavirus pneumonia. J Thromb Haemost. 2020;18(6):1421-4. https://doi.org/10.1111/ jth. 14830 .

10. Mahmoud M, Isaac N, Nadin E, Asma M, Rebecca M, Cl B. Thromboembolism risk of COVID-19 is high and associated with a higher risk of mortality: A systematic review and meta-analysis. EClinicalMedicine. 2020;29:100639. https://doi.org/10.1016/j. eclinm.2020.100639.

11. Miesbach W, Makris M. COVID-19: coagulopathy, risk of thrombosis, and the rationale for anticoagulation. Clin Appl Thromb. 2020;26:1076029620938149. https://doi.org/10.1177/1076029620 938149.

12. Rali P, O'Corragain O, Oresanya L, Yu D, Sheriff O, Weiss R, et al. Incidence of venous thromboembolism in coronavirus disease 2019: an experience from a single large academic center. J Vasc Surg Venous Lymphat Disord. 2020;9(3):585-91.e2. https:// doi.org/10.1016/j.jvsv.2020.09.006.

13. Carsana L, Sonzogni A, Nasr A, Rossi RS, Pellegrinelli A, Zerbi $\mathrm{P}$, et al. Pulmonary post-mortem findings in a series of COVID-19 cases from northern Italy: a two-centre descriptive study. Lancet Infect Dis. 2020;20:1135-40. https://doi.org/10.1016/S14733099(20)30434-5.

14. Lax SF, Skok K, Zechner P, Kessler HK, Kaufmann N, Koelblinger $\mathrm{C}$, et al. Pulmonary arterial thrombosis in COVID-19 with fatal outcome: results from a prospective, single-center, clinicopathologic case series. Ann Intern Med. 2020;173:350-61. https:// doi.org/10.7326/M20-2.

15. Rapkiewicz AV, Mai X, Carsons SE, Pittaluga S, Kleiner DE, Berger JS, et al. Megakaryocytes and platelet-fibrin thrombi characterize multi-organ thrombosis at autopsy in COVID-19: a case series. EClinicalMedicine. 2020;24: 100434. https://doi.org/10. 1016/j.eclinm.2020.100434.

16. Nougier C, Benoit R, Simon M, Desmurs-Clavel H, Marcotte G, Argaud L, et al. Hypofibrinolytic state and high thrombin generation may play a major role in SARS-COV2 associated thrombosis. J Thromb Haemost. 2020;18(9):2215-9. https://doi.org/10.1111/ jth.15016.

17. Seheult JN, Seshadri A, Neal MD. Fibrinolysis shutdown and thrombosis in severe COVID-19. J Am Coll Surg. 2020;231(2):203-4. https://doi.org/10.1016/j.jamcollsurg.2020. 05.021 .

18. Wright FL, Vogler TO, Moore EE, Moor HB, Wohlauer MV, Urban S, et al. Fibrinolysis shutdown correlation with thromboembolic events in severe COVID-19 infection. J Am Coll Surg. 2020;231(2):193-203. https://doi.org/10.1016/j.jamcollsurg.2020. 05.007 .

19. Sadd C, Rowe T, Nazeef M, Kory P, Sultan S, Faust H. Thromboelastography to detect hypercoagulability and reduced fibrinolysis in coronavirus disease 2019 acute respiratory distress syndrome patients. Crit Care Explor. 2020;2(9): e0192. https://doi.org/10. 1097/CCE.0000000000000192.
20. Zuo Y, Warnock M, Harbaugh A, Yalavarthi S, Gockman K, Zuo M, et al. Plasma tissue plasminogen activator and plasminogen activator inhibitor-1 in hospitalized COVID-19 patients. medRxiv. 2020. https://doi.org/10.1101/2020.08.29.20184358.

21. Creel-Bulos C, Auld SC, Caridi-Scheible M, Barker NA, Friend $\mathrm{S}$, Gaddh M, et al. Fibrinolysis shutdown and thrombosis in a COVID-19 ICU. Shock. 2021;55(3):316-20. https://doi.org/10. 1097/SHK.0000000000001635.

22. Hammer S, Häberle H, Schlensak C, Bitzer M, Malek NP, Handgretinger R, et al. Severe SARS-CoV-2 infection inhibits fibrinolysis leading to changes in viscoelastic properties of blood clot: a descriptive study of fibrinolysis in COVID-19. Thromb Haemost. 2021. https://doi.org/10.1055/a-1400-6034.

23. Bouck EG, Denorme F, Holle LA, Middelton EA, Blair AM, Laat B, et al. COVID-19 and sepsis are associated with different abnormalities in plasma procoagulant and fibrinolytic activity. Arterioscler Thromb Vasc Biol. 2020;41(1):401-14. https://doi. org/10.1161/ATVBAHA.120.315338.

24. Nogami K, Matsumoto T, Sasai K, Ogiwara K, Arai N, Shima M. A novel simultaneous clot-fibrinolysis waveform analysis for assessing fibrin formation and clot lysis in haemorrhagic disorders. Br J Haematol. 2019;187(4):518-29. https://doi.org/ 10.1111/bjh.16111.

25. Onishi T, Nogami K, Ishihara T, Inoue S, Kawaguchi M, Nishio $\mathrm{K}$, et al. A pathological clarification of sepsis-associated disseminated intravascular coagulation based on comprehensive coagulation and fibrinolysis function. Thromb Haemost. 2020;120(9):1257-69. https://doi.org/10.1055/s-0040-1713890.

26. McGonagle D, O'Donnell JS, Sharif K, Emery P, Bridgewood C. Immune mechanisms of pulmonary intravascular coagulopathy in COVID-19 pneumonia. Lancet Rheumatol. 2020;2(7):e437-45. https://doi.org/10.1016/S2665-9913(20) 30121-1.

27. Levi M, Ten Cate H. Disseminated intravascular coagulation. $\mathrm{N}$ Engl J Med. 1999;341(8):586-92. https://doi.org/10.1056/NEJM1 99908193410807.

28. Gando S. Microvascular thrombosis and multiple organ dysfunction syndrome. Crit Care Med. 2010;38(Suppl 2):S35-42. https:// doi.org/10.1097/CCM.0b013e3181c9e31d.

29. Shimonishi N, Ogiwara K, Oda Y, Kawabe T, Okazaki S, Shima $\mathrm{M}$, et al. A novel assessment of factor VIII activity by template matching utilizing weighted average parameters from comprehensive clot waveform analysis. Thromb Haemost. 2021;121(2):16473. https://doi.org/10.1055/s-0040-1715838.

30. Annemarie D, Ewen H, Christopher G, Hayley EH, Riinu P, Lisa N, et al. Features of 20,133 UK patients in hospital with COVID19 using the ISARIC WHO Clinical Characterisation Protocol: prospective observational cohort study. BMJ. 2020;369:m1985. https://doi.org/10.1136/bmj.m1985.

31. Christopher P, Simon J, Jie Y, Harish R, Luke O, Yelena C, et al. Factors associated with hospital admission and critical illness among 5279 people with coronavirus disease 2019 in New York City: prospective cohort study. BMJ. 2020;369: m1966. https:// doi.org/10.1136/bmj.m1966.

32. Paranjpe I, Fuster V, Lala A, Russak A, Glicksberg B, Levin M, et al. Association of treatment dose anticoagulation with in-hospital survival among hospitalized patients with COVID-19. J Am Coll Cardiol. 2020;76(1):122-4. https://doi.org/10.1016/j.jacc. 2020.05.001.

33. Rea CJ, Sorensen B, Ingerslev J, Laursen P. Fibrinogen: a procoagulant and an anticoagulant. Blood. 2011;118:384.

34. Asakura H. Classifying types of disseminated intravascular coagulation: clinical and animal models. J Intensive Care. 2014;6(2):20. https://doi.org/10.1186/2052-0492-2-20.

35. Roman W, Victor C, Wolfgang G, Michael S, Sabine Z, Marcel M, et al. Virological assessment of hospitalized patients with 
COVID-2019. Nature. 2020;581(7809):465-9. https://doi.org/10. 1038/s41586-020-2196-x.

36. Chaolin H, Yeming W, Xingwang L, Lili R, Jianping Z, Yi H, et al. Clinical features of patients infected with 2019 novel coronavirus in Wuhan, China. Lancet. 2020;395(10223):497-506. https://doi.org/10.1016/S0140-6736(20)30183-5.
Publisher's Note Springer Nature remains neutral with regard to jurisdictional claims in published maps and institutional affiliations.

\section{Authors and Affiliations}

Tomoko Onishi $^{1} \cdot$ Naruto Shimonishi ${ }^{1} \cdot$ Masahiro Takeyama ${ }^{1}$ (1) - Shoko Furukawa ${ }^{1} \cdot$ Kenichi Ogiwara ${ }^{1}$. Yuto Nakajima ${ }^{1}$ Kei Kasahara ${ }^{2} \cdot K$ Kenji Nishio ${ }^{3} \cdot K$ Kiyomi Yoshimoto ${ }^{3} \cdot$ Satoki Inoue $^{4} \cdot$ Masahiko Kawaguchi $^{4}$. Hidetada Fukushima ${ }^{5} \cdot$ Yoshihiko Saito $^{6} \cdot$ Hitoshi Yoshiji $^{7} \cdot$ Shigeo Muro $^{8} \cdot$ Kazuhiko Tsuruya $^{9} \cdot$ Sadanori Okada $^{10}$. Kazuma Sugie $^{11} \cdot$ Ryuji Kawaguchi $^{12} \cdot$ Toshiya Nishikubo $^{13} \cdot$ Masaharu Yamazaki $^{14} \cdot$ Yukio Oda $^{15} \cdot$ Toshiki Kawabe $^{15}$. Kengo Onishi ${ }^{15} \cdot$ Tomohisa Nishio $^{15} \cdot$ Keiji Nogami $^{1}$

1 Department of Pediatrics, Nara Medical University, 840 Shijo-cho, Kashihara, Nara 634-8522, Japan

2 Center for Infectious Diseases, Nara Medical University, Kashihara, Japan

3 Department of General Medicine, Nara Medical University, Kashihara, Japan

4 Department of Anesthesiology, Nara Medical University, Kashihara, Japan

5 Emergency and Critical Care Medicine, Nara Medical University, Kashihara, Japan

6 Department of Cardiovascular Medicine, Nara Medical University, Kashihara, Japan

7 Department of Gastroenterology, Nara Medical University, Kashihara, Japan

8 Department of Respiratory Medicine, Nara Medical University, Kashihara, Japan
9 Department of Nephrology, Nara Medical University, Kashihara, Japan

10 Department of Diabetes and Endocrinology, Nara Medical University, Kashihara, Japan

11 Department of Neurology, Nara Medical University, Kashihara, Japan

12 Department of Obstetrics and Gynecology, Nara Medical University, Kashihara, Japan

13 Neonatal Intensive Care Unit, Nara Medical University, Kashihara, Japan

14 Central Clinical Laboratory, Nara Medical University Hospital, Kashihara, Japan

15 SEKISUI MEDICAL CO., LTD, Tokyo, Japan 